\title{
OBSERVATIONS EN MICROSCOPIE ELECTRONIQUE A BALAYAGE SUR LA FAMILLE DES SPINTURNICIDAE (ACARINA, MESOSTIGMATA)
}

\author{
II - Ultrastructure de l'organe tarsal
}

\author{
J. DEUNFF
}

RÉSUMÉ. L'étude du complexe tarsal de quatre genres de Spinturnicidae permet de mettre en évidence des différences génériques importantes utilisables en taxonomie. La comparaison des structures observées permet de proposer un modèle fondamental du complexe tarsal avec 9 soies et une cupule centrale (renfermant 3 microsoies) chez un Spinturnicidae évolué (genre Spinturnix). 4 soies sont secrétrices d'une substance filamenteuse sortant par des microperforations située au fond de sillons creusés dans les soies. Dans cette hypothèse sur l'évolution de la famille, le genre Eyndhovenia serait moins évolué car son complexe tarsal est moins élaboré. Puis le genre Periglischrus serait encore plus primitif avec seulement une ébauche de cupule renfermant 2 microsoies. Enfin le genre le plus primitif des 4, serait le genre Paraperiglischrus qui présente seulement 7 soies et est dépourvu de cupule.

Une étude plus complète ne pourra être envisagée que lorsque nous aurons terminé les observations que nous avons entreprises dans les autres genres de la famille. Nous devrons alors tenter de comprendre les fonctions des structures observées.

\section{Ohservations with scanning on the family Spinturnicidae (Acarina, Mesostigmata). II - Ultrastructure of the tarsal organ.}

SUMMARY. The study of tarsal organ in 4 genus of the family Spinturnicidae show generic differences usable in taxonomy. It is possible to propose a fundamental model with 9 setae and a microcup containing 3 microsetae for an advanced Spinturnicidae (genus Spinturnix). Four setae secrete a substance through microhole located in furrows. In this hypothesis on the evolution of the family, the genus Eyndhovenia would be less advanced than genus Spinturnix but more than genus Periglischrus, genus Periglischrus show a sketch of microcup with only 2 microsetae. Genus Paraperiglischrus would be the most primitive with only 7 setae and without microcup.

A more complete study, will be possible when we shall have finished our observations on the other genus of the family.

Laboratoire de Parasitologie et Zoologie appliquée (Entomologie médicale), U.E.R. médicales et pharmaceutiques, Avenue du Prof. Léon Bernard, 35043 C Rennes.

Accepté le r6 septembre r98I. 


\section{Introduction}

Dans la première partie de cette étude consacrée à l'ultrastructure des Spinturnicidae (Deunff $\left.{ }^{1}\right)$, nous avons présenté les résultats préliminaires concernant la morphologie générale. Mais l'abondance des documents et la complexité, tant structurale que fonctionnelle de l'organe tarsal, nous ont conduit à en faire une étude séparée.

L'organe tarsal présent à l'extrémité de la première paire de pattes chez les acariens porte le nom d'organe de Haller chez les Ixodidae. Jusqu'à présent les principales observations en Microscopie électronique à balayage (MEB) concernent les Ixodoidea et les Rhinonyssidae (Moritsch et al., 1979). Nous présentons donc la première publication consacrée à l'ultrastructure de l'organe tarsal des Spinturnicidae en MEB. Dans cette famille les seules études de cet organe ont été réalisées en Microscopie photonique, en particulier par Neumann $(1942,1943)$ et Haarløv $(1943)$.

\section{Matériel et méthode ${ }^{2}$}

L'origine du matériel étudié et les techniques de préparation et d'observation ont été décrites dans le précédent article (Deunff, 1982). La seule modification apportée à la préparation des spécimens est la dissection préalable, dans certains cas, des pattes I. Celles-ci sont séparées à frais du reste du corps au niveau de la liaison coxae-trochanter, puis traitées comme un organisme entier.

\section{Ultrastructure de l'organe tarsal}

Notre étude porte actuellement sur quatre genres : Paraperiglischrus, Periglischrus, Eyndhovenia et Spinturnix; les autres genres sont en cours d'études et feront l'objet de prochaines publications.

Dès nos premières observations (Deunff, 1978) nous avons constaté l'abondance et la complexité morphologique des structures de l'organe tarsal. Il est situé à l'extrémité distale du tarse de la patte I en position dorsale externe. Chez les Spinturnicidae les pattes I sont orientées vers l'avant et comme dans d'autres familles d'acariens, leur rôle est probablement plutôt sensoriel que locomoteur. Nous avons d'ailleurs pu observer du matériel vivant et constater que les pattes I ne servent pas à la locomotion mais sont dirigées vers l'avant et ont des mouvements latéraux évocant ceux des antennes des insectes par exemple (Deunff, 1978).

Nous pouvons séparer les quatre genres observés en deux groupes d'après la complexité de l'organe tarsal et la présence ou l'absence d'une cupule centrale contenant des microsoies.

I. (sous presse).

2. Collaboration technique : J. Le Lannic, Centre Technique de Microscopie Électronique à Balayage de Rennes. 

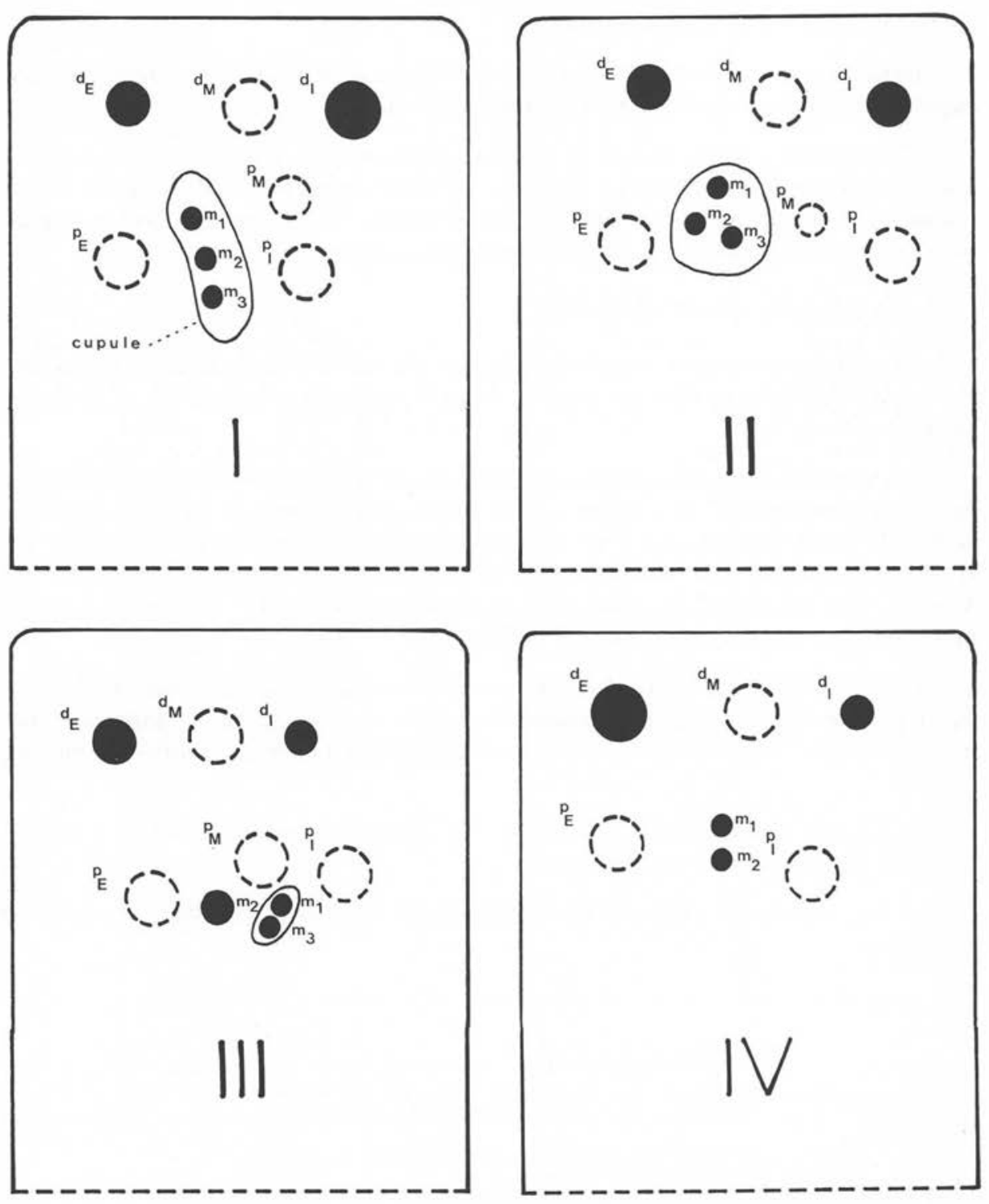

Planche I

Chaetotaxie comparée de l'organe tarsal de quatre genres de Spinturnicidae : I Spinturnix, II Eyndhovenia, III Periglischrus, IV Paraperiglischrus. 
Dans le type " simple " nous trouvons les genres Paraperiglischrus (nombre de soies réduit et absence de cupule centrale) et Periglischrus (ébauche de cupule centrale contenant deux microsoies).

Dans le type " complexe " les genres Eyndhovenia et Spinturnix présentent une cupule centrale bien nette contenant trois microsoies).

En revanche aucune différence morphologique notable n'a été observée entre les mâles et les femelles à ce niveau. L'organe tarsal ne présenterait donc pas de dimorphisme sexuel. De même les quelques observations faites sur des deutonymphes montrent des structures semblables à celles des adultes.

Les genres Eyndhovenia et Spinturnix

Le complexe sensoriel tarsal est situé sur une excroissance de la région latérodorsale du tarse I. Les soies au nombre de neuf sont très polymorphes et disposées en deux séries.

Les symboles utilisés représentent uniquement la position a natomique des soies En effet, actuellement, il n'existe aucune étude satisfaisante de la chaetotaxie de la patte I des Spinturnicidae. Une nomenclature correcte ne peut être proposée que si elle tient compte de l'ensemble de la famille ou mieux encore de l'ensemble des Mesostigmata. En attendant, nous utilisons les abréviations : $\mathrm{d}=$ distale, $\mathrm{p}=$ proximale, $\mathrm{M}=$ médiane, $\mathrm{E}=$ externe, $\mathrm{I}=$ interne, $\mathrm{m}=$ microsoie.

Dans la série distale : $d_{E}, d_{M}$ et $d_{I}$ sont alignées. Dans la série proximale : $p_{E}$, $\mathrm{p}_{\mathrm{M}}$ et $\mathrm{p}_{\mathrm{I}}$ sont soit alignées (g. Eyndhovenia), soit en triangle (g. Spinturnix). Les microsoies sont toujours situées dans le groupe proximal et seront notées $\mathrm{m}_{1}, \mathrm{~m}_{2}, \mathrm{~m}_{3}$ (Pl. I).

- La soie $d_{E}$ est longue, fine, recourbée à son extrémité. Sa surface est lisse. Elle a probablement un rôle tactile.

- La soie $d_{r}$ est plus courte, de plus forte section et rectiligne, sans relief particulier.

Planche II. - Spinturnix myoti :

I) Mâle : organe tarsal gauche, vue d'ensemble latéro-dorsale X 300 .

2) Femelle : organe tarsal gauche, détail de la cupule centrale présentant un éperon latéral $\mathrm{X} 2400$.

3) Mâle : organe tarsal droit, vue d'ensemble antéro-dorsale $\mathrm{X}$ zoo.

4) Mâle : organe tarsal gauche, vue de profil X $55^{\circ}$.

5 Femelle : organe tarsal gauche, détail de la cupule centrale $\mathrm{X} 2200$.

6) Mâle : organe tarsal droit, vue d'ensemble dorsale X $45^{\circ}$.

7) Mâle : organe tarsal gauche, vue latéro-dorsale X 550 .

8) Mâle : organe tarsal gauche, détail de la cupule centrale en vue dorsale X 2000.

9) Femelle : organe tarsal droit, vue de profil montrant les quatre soies « sécrétrices « $\mathrm{X} 900$.

ro) Femelle : organe tarsal gauche, détail d'une soie " sécrétrice " $\mathrm{X} 3500$.

II Femelle : organe tarsal droit, détail de la cupule centrale et d'une soie « sécrétrice " $\mathrm{X} 2500$.

12) Femelle : organe tarsal gauche, détail d'une soie " non sécrétrice » $\mathrm{X} 6500$. 

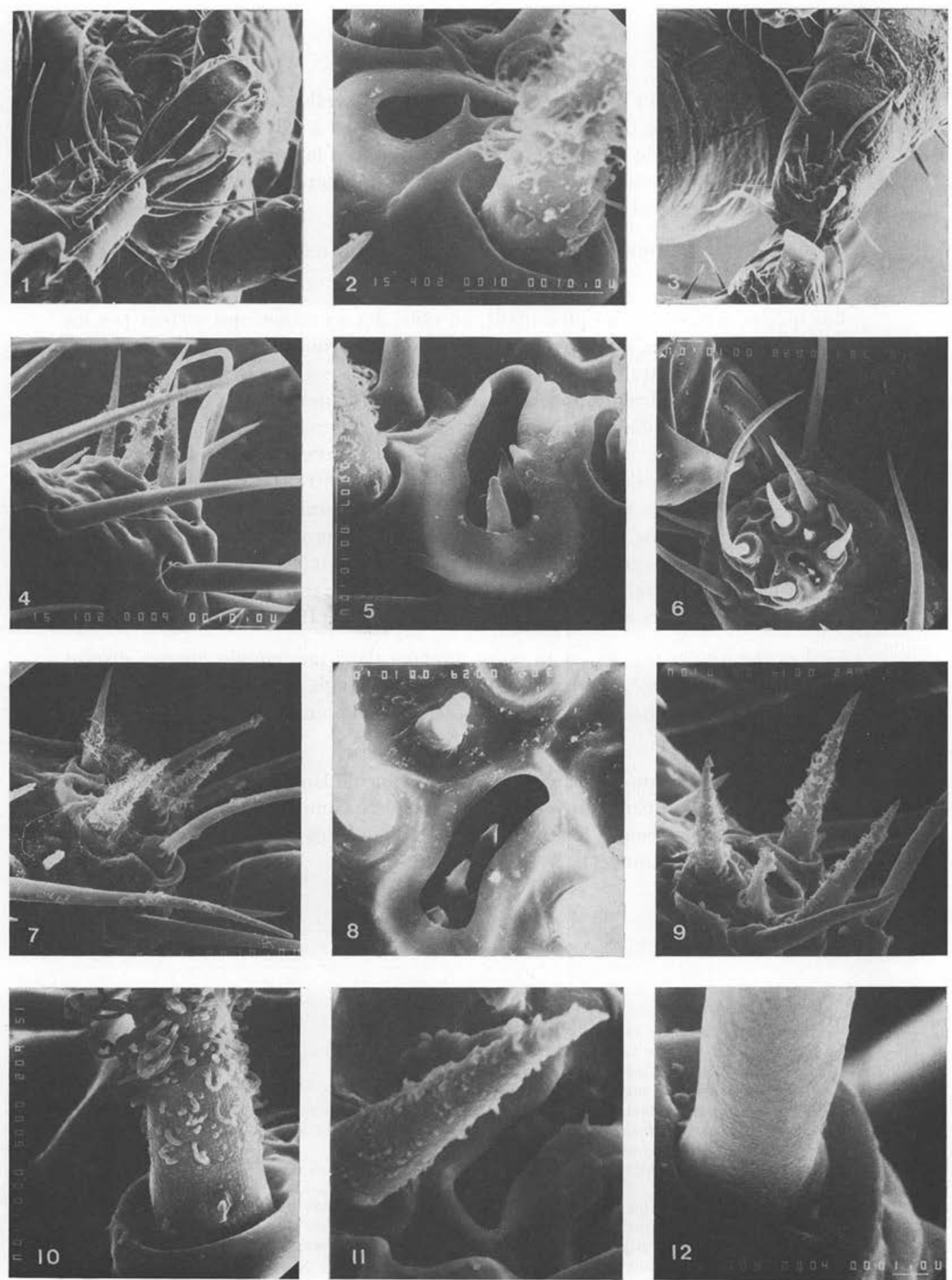

Planche II 
- Les soies $d_{M}, p_{E}$ et $p_{1}$ sont très semblables entre elles, de forte section, leur taille est proche de celle de $d_{r}$. Mais leur relief est tout à fait caractéristique : on distingue tout d'abord de fins sillons qui creusent toute la surface de la soie. Puis au fond, à des grossissements supérieurs à 5000 , on notre la présence de microperforations régulièrement espacées.

- La soie $\mathrm{p}_{\mathrm{M}}$ est courte et de faible section et son relief est identique à celui de $d_{\mathrm{M}}, \mathrm{p}_{\mathrm{E}}$ et $\mathrm{p}_{\mathrm{I}}$.

Ces quatre dernières soies présentent, en plus, des secrétions qui sortent par les microperforations comme au travers de " filières " et forment des pseudo-filaments plus ou moins denses autour de ces soies. Le rôle de ces secrétions est actuellement inconnu. Le grand nombre d'observations (600 microphotos) dans des genres différents permet d'éliminer l'hypothèse de l'artefact. Il s'agirait donc d'une substance pouvant avoir un rôle de messager (phéromone ?). Ces secrétions ont été observées chez les mâles et les femelles mais les molécules peuvent être très différentes tout en présentant des propriétés physiques et donc un aspect semblable. On ne peut donc écarter l'hypothèse d'une secrétion à ce niveau de phéromone sexuelle seule ou associée à d'autres substances. A posteriori nous avons constaté d'ailleurs que le maximum de secrétion était semble-t-il présent en période estivale, période de reproduction de la plupart des espèces (Deunff et Beaucournu, 1981).

- Les microsoies $m_{1}, m_{2}$ et $m_{3}$ sont alignées dans une cupule longue, étroite et profonde dans le genre Spinturnix et disposées en triangle dans une cupule presque circulaire et peu profonde dans le genre Eyndhovenia, elle sont effilées à leur extrémité (Pl. II et III).

Cette structure évoque fortement celle de l'organe de Haller des Ixodidae (Aeschlimann, communication personnelle) que l'on considère comme un système récepteur olfactif essentiellement. Seule l'histologie, l'histochimie et les études de comportement pourront confirmer ou non cette hypothèse.

\section{Planche III}

I) Spinturnix psi $\widehat{0}$, organe tarsal droit, vue dorsale $\mathrm{X} 600$.

2) S. emarginatus 0 , organe tarsal droit, vue dorsale $\mathrm{X} 600$.

3) S. mystacinus $\sigma^{+}$, organe tarsal droit, vue dorsale $\mathrm{X} 600$.

4) Eyndhovenia euryalis $\sigma^{-}$, organe tarsal droit, vue dorsale X 650 .

5) E. euryalis +, organe tarsal droit, détail d'une soie striée et microperforée $\mathrm{X} 9500$.

6) E. euryalis $\hat{\sigma}$, organe tarsal gauche, détail de la cupule centrale $\mathrm{X} 2600$.

7) S. mystacinus $\sigma^{\top}$, organe tarsal droit, vue de profil $\mathrm{X} 600$.

8) Paraperiglischrus rhinolophinus $\widehat{\sigma}$, organe tarsal gauche, détail d'une soie microperforée et " sécrétrice " $\mathrm{X} 5000$.

9) P. rhinolophinus , organe tarsal gauche, vue d'ensemble antéro-dorsale $\mathrm{X} 600$.

Io) $P$. rhinolophinus $\widehat{\delta}^{\text {, }}$ organe tarsal gauche, vue d'ensemble dorsale $\mathrm{X} 950$.

I I) Periglischrus iheringi ô, organe tarsal droit, vue d'ensemble dorsale X r 200.

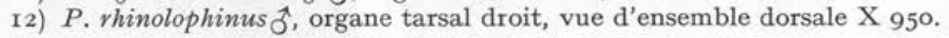



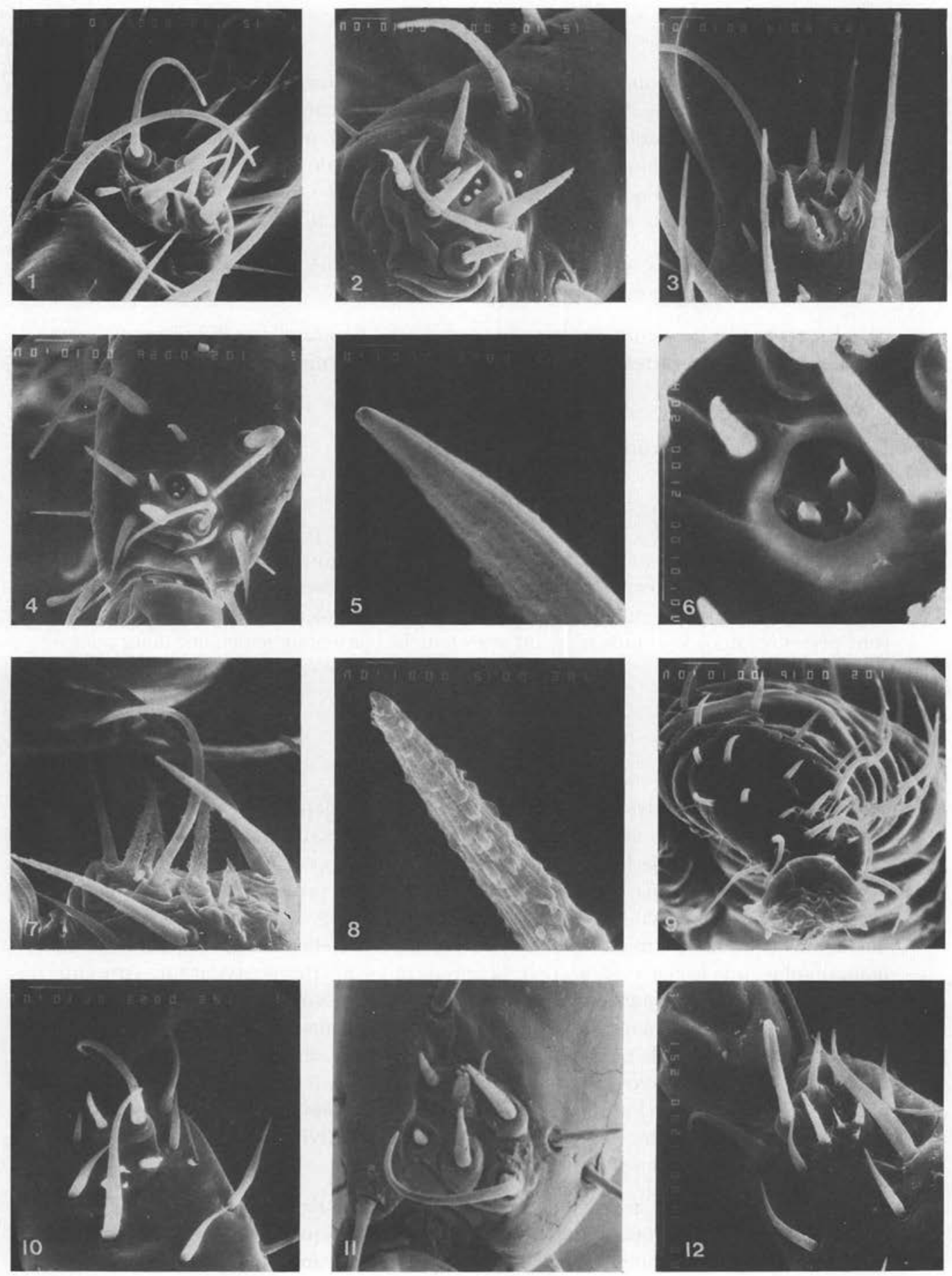

Planche III 
La structure du complexe tarsal semble assez constante au sein d'un même genre. Ainsi entre $S$. myoti, S. mystacinus et $S$. emarginatus nous n'avons noté que des variations de très faible amplitude : chez $S$. myoti la bordure de la cupule centrale présente un éperon; la lumière de la cupule est allongée, de largeur presque constante et les microsoies sont alignées. Chez $S$. emarginatus la cupule est légèrement triangulaire, les microsoies ne sont plus alignées, l'éperon est absent ( $P l$. III).

Chez S. mystacimus nous avons seulement remarqué, par rapport à $S$. myoti, l'absence d'éperon en bordure de la cupule.

En revanche les structures tarsales des genres Spinturnix et Eyndhovenia sont très différentes et confirment encore la validité de ce dernier genre créé par Rudnick en 1960.

\section{Les genres Paraperiglischrus et Periglischrus}

La disposition et la structure des soies présentent de nombreuses analogies avec les genres précédents, les soies $\mathrm{d}_{\mathrm{E}}$ et $\mathrm{d}_{\mathrm{I}}$ sont lisses, $\mathrm{d}_{\mathrm{M}}, \mathrm{p}_{\mathrm{E}}$, $\mathrm{p}_{\mathrm{I}}$ et $\mathrm{p}_{\mathrm{M}}$ sont striées et microperforées. Mais on note aussi des différences importantes au niveau du complexe tarsal. Dans le genre Paraperiglischrus, deux soies sont absentes ( $\mathrm{p}_{\mathrm{M}}$ et une mícrosoie) et il n'y a pas de cupule centrale. Dans le genre Periglischrus, les neuf soies du complexe sont présentes mais la cupule n'est qu'une ébauche contenant seulement deux microsoies (Pl. III).

\section{Discussion}

L'étude de ces quatre genres, bien que très incomplète, permet de proposer un schéma de la structure fondamentale du complexe tarsal chez les Spinturnicidae. Si l'on accepte en partie la classification évolutive proposée par Rudnick, le genre Spinturnix serait le plus évolué. Nous pouvons donc présenter comme structure évoluée de l'organe tarsal, le " type Spinturnix " avec : 9 soies dont 4 secrétrices et une cupule contenant 3 microsoies. Dans cette hypothèse le genre Eyndhovenia serait moins évolué, que le genre Spinturnix, la cupule et les microsoies ayant une structure moins élaborée. En revanche Eyndhovenia serait plus évolué que Periglischrus, ce qui n'est pas en accord avec l'hypothèse de Rudnick. Dans le genre Periglischrus on trouverait donc un stade encore plus primitif avec bien sûr 9 soies, mais seulement une ébauche de cupule avec 2 soies au lieu de 3. L'origine de la formation de la cupule pourrait être la fusion des aires d'insertion de deux soies puis d'une troisième ou encore une division d'une soie en deux, l'une d'elle se divisant à nouveau en deux, dans une même aire d'insertion.

Il est actuellement impossible de conclure, la première hypothèse semble plus plausible en supposant que peu à peu les soies périphériques viennent s'intégrer au complexe tarsal. Malheureusement l'observation ne confirme pas cette hypothèse 
car quelque soit le nombre des soies du complexe, la position et le nombre des soies périphériques semblent peu varier.

Enfin le genre le plus primitif parmi les 4 étudiés, pourrait être Paraperiglischrus qui possède seulement 7 soies et ne présente pas de cupule. Dans ce cas l'évolution de cette structure sensorielle irait vers une augmentation du nombre des soies spécialisées et vers une complexification (système cupule-microsoies) de l'organe tarsal. Cette évolution irait donc à l'inverse de la régression morphologique due au parasitisme. On constate une adaptation et une spécialisation de ces acariens à leur mode de vie très particulier sur les membranes alaires des Chiroptères. Ces faits sont en accord avec les résultats de Fain $(1969,1977,1979,1981)$ concernant l'adaptation des acariens au parasitisme.

Il est en fait encore trop tôt pour proposer une véritable étude phylogénique des Spinturnicidae. Il faut au préalable étudier parfaitement les dix genres actuellement connus avant de tenter une véritable synthèse de nos connaissances. La valeur du complexe tarsal comme critère évolutif reste d'ailleurs à préciser.

\section{Conclusion}

L'étude préliminaire de l'organe tarsal de quatre genres de Spinturnicidae permet de mettre en évidence des différences génériques importantes pouvant être utilisées en taxonomie. Nous avons tenté de relier ces structures à l'évolution de ces acariens parasites. Toutefois cette étude ne sera complète que lorsque nous aurons terminé nos observations actuellement en cours sur les autres genres de la famille. Il sera alors nécessaire pour comprendre les structures observées, d'en faire l'étude histologique, biochimique et neurophysiologique, tous ces résultats devant être confrontés aux faits écologiques et éthologiques.

Remerciements. Nous adressons nos vifs remerciements aux Professeurs Fain, Aeschlimann et Allégret, au Docteur Gaud et tout particulièrement au Professeur Beaucournu pour leurs précieux conseils.

\section{BIBLIOGRAPHIE}

DEunfF J. : Contribution à l'étude de la famille des Spinturnicidae (Acarina, Mesostigmata) de la région paléarctique occidentale. II. Données sur la morphologie et la systématique des Spinturnicidae. Mémoire de D. E. A., Université de Rennes, 1978 , 32, 17 pl.

DEUNFF J. : Observation en microscopie à balayage sur la famille des Spinturnicidae (Acarina, Mesostigmata). I. Morphologie générale. Acarologia, 1982, (sous presse).

Deunff J., Beaucournu J. C. : Phénologie et variations du dermecos chez quelques espèces de Spinturnicidae (Acarina, Mesostigmata). Ann. Parasitol. Hum. Comp., I981 56, 203-224.

FArN A. : Adaptation to parasitism in mites. Acarologia, 1969, II, 429-449.

FAIN A. : Observations sur la spécificité des Acariens de la famille Myobiidae. Corrélation entre l'évolution des parasites et de leurs hôtes. Ann. Parasitol. Hum. Comp., 1977, S2, 339-351. 
FArN A. : Specificity, adaptation and parallel host-parasite evolution in acarines, especially myobiidae, with a tentative explanation for the regressive evolution caused by the immunological reactions of the host. In : Recent Advances in Acarology, Academic Press, London, 1979, vol. 2, 32I-328.

Farn A. : Spécificité et évolution parallèle hôtes-parasites chez les Myobiidae (Acari). Deuxième Symposium sur la spécificité parasitaire des parasites de Vertébrés, Paris, I3-17 avril r981.

HAARLøV N. : The chaetotaxis of tarsus I in some mesostigmata. With special reference to a supposed homologon of the tarsal organ of the genus Spinturnix and Haller's organ of the ticks. Ent. Medd. Kobenttavn, 1943, 23, 273-294.

Moritsch Ch., Sixl-Voigt B., Sixl W., FAin A. : Das sinnesfeld am tarsus I bei nasalen milben am beispiel von Mesonyssus columbae und vergleichend Mesonyssus melloi, Proc. 4 th Int. Congress Acarol., 1979, 717-723.

Neumann K. W. : Besitzen die mesostigmata ein dem Hallerschen der zecken Homologes tarsalorgan? Z. Morph. Okol. tiere, $194239,47-75$.

Neumann K. W. : Noch einmal : Das tarsalorgan bei Spinturnix v. Heiden. Zool. Anz. Leipzig, I943, 143 , I05-II6.

RudNICK A. : A revision of the mites of the family Spinturnicidae (Acarina). Univ. Calif. Publ. Ent., I960, $17, \mathrm{I}_{57-284}$. 